\title{
Bioluminescence Imaging of Angiogenesis in a Murine Orthotopic Pancreatic Cancer Model
}

\author{
Eliane Angst, ${ }^{1,3,4}$ Monica Chen, ${ }^{1}$ Michelle Mojadidi, ${ }^{1}$ O. Joe Hines, ${ }^{1,2}$ \\ Howard A. Reber, ${ }^{1,2}$ Guido Eibl ${ }^{1,2}$
}

\author{
${ }^{1}$ Hirshberg Laboratory for Pancreatic Cancer Research, Department of Surgery, UCLA Center for Excellence in Pancreatic Diseases, \\ David Geffen School of Medicine at UCLA, Los Angeles, CA, USA \\ ${ }^{2}$ UCLA Jonsson Comprehensive Cancer Center, Los Angeles, CA, USA \\ ${ }^{3}$ Department of Visceral and Transplant Surgery, Inselspital, University of Bern, Bern, Switzerland \\ ${ }^{4}$ Department of Surgery, David Geffen School of Medicine at UCLA, 675 Charles E. Young Drive South, MRL 2535, Los Angeles, CA \\ 90095, USA
}

\begin{abstract}
Purpose: Angiogenesis is essential for physiological processes as well as for carcinogenesis. New approaches to cancer therapy include targeting angiogenesis. One target is VEGF-A and its receptor VEGFR2. In this study, we sought to investigate pancreatic cancer angiogenesis in a genetically modified VEGFR2-luc-KI mouse.

Procedures: Live in vivo bioluminescence imaging of angiogenesis was performed continuously until sacrifice in subcutaneous tumors as well as in orthotopically transplanted tumors. Tumor tissue was immunostained for CD-31 and VEGFR2.

Results: Peritumoral angiogenesis measured by light emission was detected beginning at week 3 following subcutaneous injection. In the orthotopic model, light emission began at day 4 , which likely corresponds to wound healing, and continued throughout the experimental period during tumor growth. Peritumoral CD-31 vessel- and VEGFR2-staining were positive.

Conclusions: The VEGFR2-luc-KI mouse is a valuable tool to demonstrate tumor angiogenesis and seems to be suitable to evaluate anti-angiogenic approaches in pancreatic cancer.
\end{abstract}

Key words: Pancreatic cancer, Angiogenesis, Bioluminescence, VEGFR2

\section{Introduction}

A ngiogenesis represents the formation of new capillaries by the outgrowth from existing microvessels. While angiogenesis is essential for most physiological processes, e.g., embryonic development, menstrual cycle, and wound repair, unregulated angiogenesis is involved in many pathological conditions, including cancer. Tumor formation is a complex process involving cell proliferation and cell death. In fact,

Brief article: this work is significant to basic researchers studying angiogenesis in pancreatic cancer

Correspondence to: Eliane Angst; e-mail: eliane.angst@gmail.com tumors cannot grow larger than a few millimeters without the assembly of new blood vessels to supply the necessary nutrients and oxygen. It is now clear that progression of tumor growth and metastasis are angiogenesis dependent $[1,2]$. This phenomenon is called the angiogenic switch, which is dependent on the balance between pro-angiogenic and anti-angiogenic factors expressed by the tumor and the stromal cells. Failure of a tumor to recruit new microvascular endothelial cells or to reorganize the existing surrounding vasculature results in a non-angiogenic tumor that is only microscopic in size (less than $1 \mathrm{~mm}^{3}$ ), as the diffusion limit of oxygen is approximately $100 \mu \mathrm{m}$ through tissue [2]. Small tumors are highly dependent on their microenvironment for oxygen and the supply of nutrients, whereas macroscopic tumors bear well-organized vascular structures filled with red blood cells [2]. In contrast to 
normal vessels, tumor vascular structures are disorganized and leaky.

Angiogenesis is a complex biological process that involves a cascade of intra- and extracellular events. Vascular endothelial growth factor (VEGF) is a very potent and well-studied inducer of angiogenesis. Stimulated endothelial cells produce proteases that degrade the surrounding basement membrane. Endothelial cells and vascular smooth muscle cells proliferate and migrate to align and form new vessels. The endothelial cells produce specific growth factors, such as platelet-derived growth factor and transforming growth factor beta, to attract the supporting pericytes. A new specific basement membrane is subsequently produced resulting in a mature vessel [3].

Proliferating endothelial cells in vessels undergoing active angiogenesis express receptors for VEGF (VEGFR). Three different VEGFRs have been identified and shown to be sensitive to VEGF ligands. VEGFR1 and VEGFR2 are expressed on vascular endothelial cells, but the biological angiogenic activity is mainly mediated through VEGFR2 [3]. VEGFR3 is expressed on lymphatic endothelial cells and receptor signaling induces lymphangiogenesis. Several ligands bind and activate human VEGFR2, including VEGF-A, processed VEGF-C and VEGF-D, VEGF isolated from snake venom as well as VEGF-like proteins encoded by parapox virus open reading frames (VEGF-E family). Murine VEGF-D fails to bind to VEGFR2. Both human VEGF-D and VEGF-C bind to VEGFR2 with an affinity one order of magnitude weaker than VEGF-A. Therefore, the angiogenic activity is mainly mediated through VEGFR2 and VEGF-A [4]. Targeted inactivation of either VEGF-A or VEGFR2 interferes with angiogenesis. Indeed mouse embryos lacking VEGF-A or VEGFR2 die at 8.5 or 9.5 embryonic days, respectively [5]. In tumors, the levels of VEGFR2 mRNA are significantly increased compared to normal tissues [6]. VEGF-A binding to VEGFR2 induces proliferation, migration, and differentiation of vascular endothelial cells [4]. VEGFR2 signaling encompasses a variety of coordinated intracellular events. The activation of PI3K/Akt pathway leads to cell survival while activation of the MEK/ERK pathways appears to be crucially involved in mediating signals for endothelial cell proliferation. Activation of FAK and paxillin via tyrosine phosphorylation mediated by VEGFR 2 also leads to recruitment of actin-anchoring proteins - events thought to be essential for vascular endothelial cell migration [7].

Newer approaches to cancer therapy involving the blockade of angiogenesis represent a promising research field $[1,8]$. But methods to study angiogenesis in vivo with longitudinal measurements over time are cumbersome and limited, as it usually requires the sacrifice of the animal and visualization of microvessels by immunohistochemical staining of endothelial cell-specific markers. Novel and less invasive approaches to study angiogenesis are needed in preclinical studies to advance this field.

Therefore, we sought to use a murine angiogenesis model which enables us to monitor VEGFR2 promoter activity. We used the approach of genetically modified animals in which the VEGFR2 promoter drives the expression of luciferase and thus allows direct visualization of angiogenesis using bioluminescence in vivo. We show here, for the first time, the feasibility to image tumor angiogenesis with the genetically modified VEGFR2-luc-KI mouse in an orthotopic pancreatic cancer model.

\section{Materials and Methods}

\section{Reagents}

Rat anti-mouse PECAM-1 (CD-31) antibody was purchased from Angio-Proteomie (mAP-0032, Boston, MA, USA), and rabbit antiVEGFR2 antibody was from Cell Signaling Technology, Inc. (Danvers, MA, USA). Heterozygous VEGFR2-luc-KI mice with a $\mathrm{nu} / \mathrm{nu}$ background were obtained from Xenogen, Caliper Life Sciences, Inc. (Hopkinton, MA, USA). These mice were created using a gene-targeting approach that "knocked in" firefly luciferase cDNA into the first exon of the endogenous VEGFR2 locus in murine $129 / \mathrm{SvEv}$ embryonic stem cells. Luciferin was provided by the Crump Institute for Molecular Imaging at UCLA.

\section{Orthotopic xenograft}

Animal studies were approved by the Chancellor's Animal Research Committee of the University of California, Los Angeles, in accordance with the National Institutes of Health Guide for the Care and Use of Laboratory Animals. The orthotopic xenograft was performed as described earlier [9]. Briefly, $2 \times 10^{6}$ HPAF-II pancreatic cancer cells were injected subcutaneously into the flank of two anesthetized donor nude mice. The donor tumors were measured at the time of imaging in two dimensions. Tumor surface was calculated from the length and the width with the formula (length $/ 2) \times($ width $/ 2) \times \pi$. After 4 weeks, the donor tumor was harvested and minced to fragments of approximately $1 \mathrm{~mm}^{3}$. Only macroscopically viable tissue from the periphery of the subcutaneous tumor was used for the orthotopic transplantation. Three recipient nude mice were anesthetized with isoflurane and opened by a left longitudinal laparotomy. The spleen, together with the pancreatic tail, was gently exteriorized, and a tissue pocket was created in the pancreatic parenchyma. A tumor fragment was placed into the tissue pocket so it was entirely surrounded by normal pancreas. After careful relocation of the pancreas and spleen into the abdominal cavity, the abdominal wall was closed in two layers. Mice were imaged in regular intervals (see below). After 6 weeks, the animals were imaged before and after sacrifice and evaluated for tumor, ascites, and metastasis. Samples were preserved as freshly frozen and in $10 \%$ formalin before processing.

\section{Imaging}

Imaging and recording was performed at days $15,22,25$, and 28 after the subcutaneous injection and at days 4, 8, 14, 23, 30, 37, and 45 after tumor implantation into the orthotopic position at the Imaging Core Facility at UCLA. After anesthesia with isoflurane, the mice were injected intraperitoneally with $3 \mathrm{mg}$ luciferin. In the first week, we showed in a time-course experiment over 30 min that the light emission was stable $10 \mathrm{~min}$ after the injection. At each day 
of imaging, several measurements were taken between 10 and 25 min after injection. Imaging and data processing were performed in an IVIS ${ }^{\text {TM }} 100$ Imaging System with the Living Image $\AA 2.50 .1$ software: a photographic image was taken and then the light acquisition was performed for bioluminescence. Both pictures were superimposed. Light intensity was quantified in the region of interest. Output was expressed in photons $/ \mathrm{s} / \mathrm{cm}^{2} /$ steridian. Three to four measurements were averaged for each animal. Data are presented as mean \pm standard deviation (SD) of the measurement at each time point.

\section{Immunohistochemistry}

Four-micrometer cryostat sections were fixed in acetone. Endogenous peroxidase activity was blocked in 3\% hydrogen peroxide and background was blocked with $20 \%$ normal serum (from the same animal species as the secondary antibody) for $1 \mathrm{~h}$ at room temperature. The sections were incubated with the anti-CD-31 antibody for $2 \mathrm{~h}$ at room temperature, including negative controls that were performed by omission of the primary antibody. The biotinylated secondary antibody was followed by components of the Vectastain ${ }^{\circledR}$ Kit (Vector Laboratories Inc., Burlingame, CA, USA) according to the manufacturer's instruction. Immunoreactivity was developed using 3,3'-diaminobenzidine as the peroxidase substrate and nuclei were counterstained with hematoxylin. A similar technique was applied for VEGFR2 staining on paraffin sections except for antigen retrieval in citrate buffer, incubation of the sections with the anti-VEGFR2 antibody overnight at $4{ }^{\circ} \mathrm{C}$, and an isotype $\operatorname{IgG}$ for negative control. Serial sections were stained with hematoxylin and eosin and documented using a Nikon Eclipse 90i microscope and NIS-Elements AR 3.0 software from Nikon Instruments Inc. (Melville, NY, USA).

\section{Results}

To assess whether we could visualize tumor angiogenesis after subcutaneous injection of pancreatic cancer cells, the donor mice were imaged after the initial appearance of a macroscopically visible tumor after day 14. Although the tumor started to be visible and palpable, light emission in the peritumoral region stayed at background values (Fig. 1). These animals have a relatively high background of VEGFR2-promoter activity, corresponding to light emission of about $3.5 \times 10^{6}$ photons $/ \mathrm{s} / \mathrm{cm}^{2} /$ steridian. We found even higher unstimulated light emission in the testicles, presumably intra-abdominal reproductive organs, liver, and spleen. Consistent with these results VEGFR-2 has been found to be expressed in the Leydig cells of the testicles and in normal hepatic tissue [10,11]. Spleen and liver bear an intricate network of vessels requiring constant remodeling of the capillaries which may account for the observed VEGFR2 promoter activity. Over the next 2 weeks, there was a 2.7 fold increase in light emission at the tumor site $\left(9 \times 10^{6}\right.$ photons $/ \mathrm{s} / \mathrm{cm}^{2} /$ steridian). The measured elevation in light emission paralleled the increase of the tumor surface area (Fig. 1, inset).

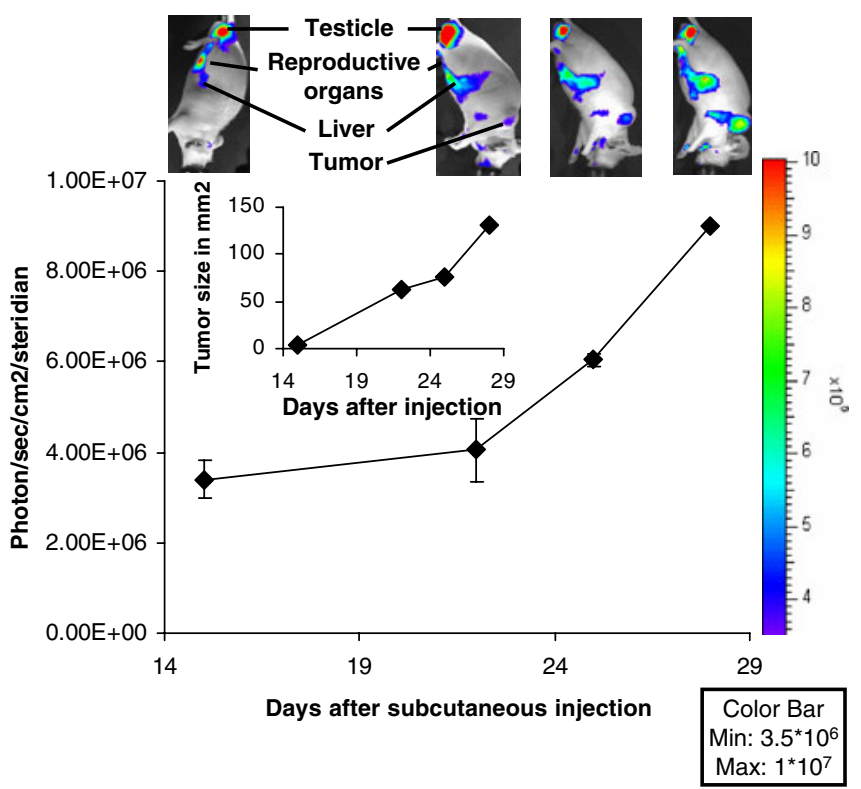

Fig. 1. Imaging of a subcutaneous donor tumor. Male donor VEGFR2-luc-KI mice were injected with $2 \times 10^{6}$ cells subcutaneously. They were imaged as described in "Materials and Methods" section. These mice have a relatively high background of VEGFR2 promoter activity and specific activity in the testicles, the intra-abdominal sexual organs, spleen, and liver. There was a steady increase in light emission at the tumor site over time. The insert shows the increase in tumor area $\left(\mathrm{mm}^{2}\right)$. Error bars represent the SD of the measurement at each time point.

After tumor implantation in the orthotopic position, the animals were imaged at day 4 and a specific light emission at the site of implantation about 3-fold over background values was already observed $\left(1.1 \times 10^{7}\right.$ photons $/ \mathrm{s} / \mathrm{cm}^{2} / \mathrm{steri}$ dian; Fig. 2). The light emission peaked at day $8\left(1.5 \times 10^{7}\right.$ photons $/ \mathrm{s} / \mathrm{cm}^{2} /$ steridian) and then returned to initial values at day 14. This first peak most likely corresponded to wound healing. Accordingly, after surgery the abdominal wound had initially a fibrinous coating and was completely healed at day 14. After day 14, the light emission increased again gradually at the site of implantation with a second peak at day $30\left(2.1 \times 10^{7}\right.$ photons $/ \mathrm{s} / \mathrm{cm}^{2} /$ steridian $)$. Thereafter, the light signal decreased with a final value of $1.4 \times 10^{7}$ photons/ $\mathrm{s} / \mathrm{cm}^{2} /$ steridian at day 45 . Overall, the specific light emission at the implantation site was about 2-fold higher at day 30 compared to day 4, suggesting locally elevated VEGFR2 promoter activity and angiogenesis at the tumor site. In all the measurements, the small variation between sampled images indicates stable light emission over time.

After 6 weeks, each animal was individually imaged preand post-mortem. Luciferin was injected and light emission in the anesthetized living animal was measured. Then a final laparotomy was performed and the orthotopically grown tumors were removed. In one mouse, a tumor ventral to the spleen and a second tumor growing orthotopically in the tail of the pancreas was found (Fig. 3). Both tumors and the skin covering the tumors were removed. The animals were 


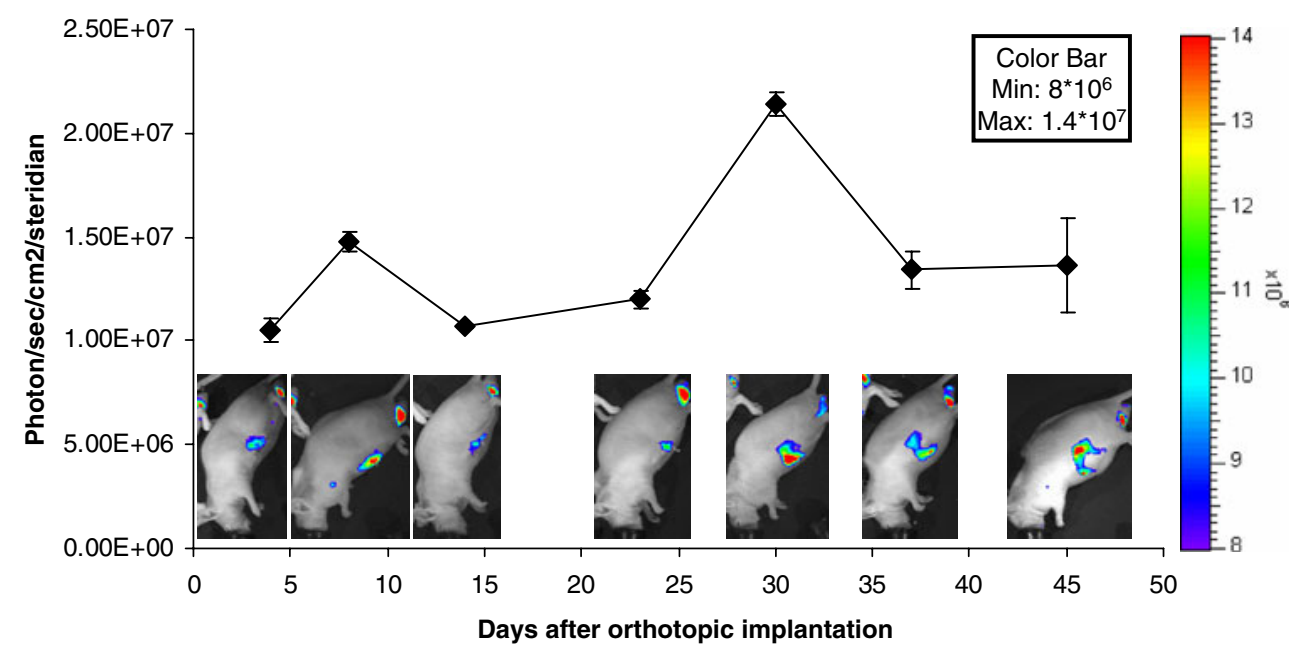

Fig. 2. Imaging of an orthotopic tumor. Pieces of the donor tumors were transplanted into male recipient VEGFR2-luc-KI mice, which were imaged weekly. Localized light emission was detected. Error bars represent the SD of the measurement at each time point.

euthanized and all removed parts were separately imaged again. After tumor harvest, only the background level of $3.5 \times 10^{6}$ photons $/ \mathrm{s} / \mathrm{cm}^{2} /$ steridian, corresponding to $26 \%$ of the initial light emission, remained in the euthanized animal, indicating the removal of all the tissue with induced luciferase activity. Separate measurements demonstrated strong luciferase activity in the first ventral tumor $(86 \%$ of the initial measurement) and the second orthotopic tumor $(56 \%)$. The skin covering the intra-abdominal tumors only accounted for $4 \%$ of the initial light emission. Addition of light emission from the different parts after sacrifice is larger than the total light emission before sacrifice due to the absorbance caused by the overlaying tissue in the live

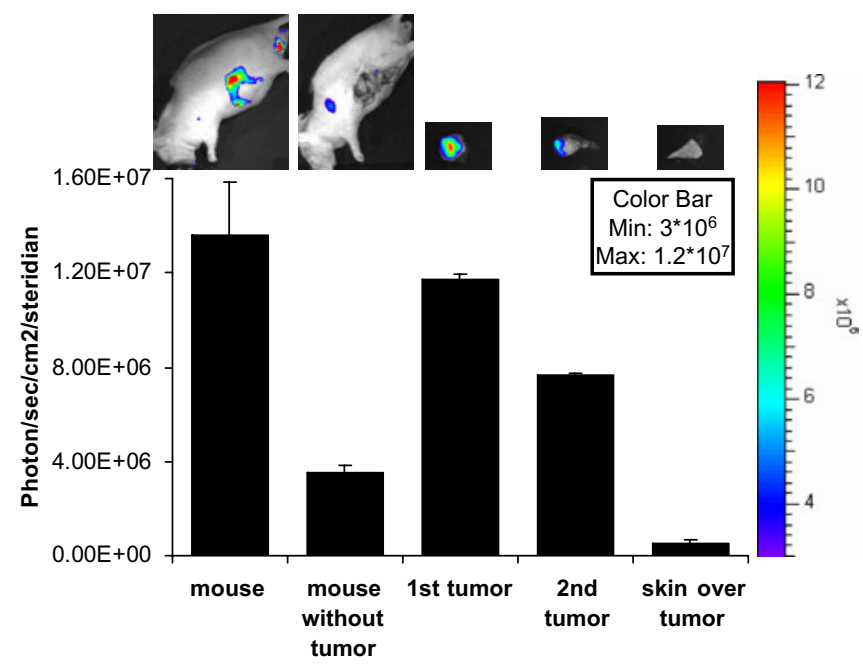

Fig. 3. Imaging at sacrifice. After 6 weeks of tumor growth, the animals were imaged in vivo and post-mortem. The animal, tumors, and the skin which covered the tumors were imaged and quantified separately. In the post-mortem imaging, the total light emission is stronger because no light is absorbed through the tissue. Error bars represent the SD of the measurement at each time point. animal. We found locally growing tumors without any apparent ascites and no distant metastasis in all the animals.

To assess whether the measured light emission corresponded to the presence of blood vessels, frozen tissue was processed and stained for the endothelial cell marker CD-31 (Fig. 4). Immunohistochemical staining for CD-31 not only showed mainly peritumoral vessels but also intratumoral vessels within the stromal tissue between cancer cells. In addition, VEGFR2, another marker of endothelial cells, was found to be faintly expressed on peritumoral vessels (Fig. 4).

\section{Discussion}

The current study demonstrates the feasibility to monitor angiogenesis in longitudinal measurements in a VEGFR2luc-KI mouse applying an orthotopic pancreatic cancer model. Due to the low background activity of the VEGFR2 promoter in the pancreas, this mouse is suited to study local tumor growth with its accompanying angiogenesis and wound healing. In contrast, a metastatic model would be difficult to investigate for the high background activity found in several other organs.

In vivo analysis of tumor angiogenesis is a crucial step to evaluate therapeutic anti-angiogenic strategies and the involved regulatory pathways. Histological and molecular techniques allow analyses on the subcellular and molecular level. However, they are not suitable for longitudinal functional studies. Intravital microscopy has a high spatial resolution, but the necessary tissue preparation affects the physiology of angiogenesis and the depth of imaging is only several hundred microns within living tissue [12]. This is not feasible for the pancreas, which lies in the depth of the abdomen. Non- or minimally invasive imaging techniques can obtain temporal dynamics and quantitatively determine physiological functions. VEGFR2 imaging has been performed previously with success, particularly in angiogenesis-directed treatment studies [13-16]. Contrast-enhanced 
H\&E

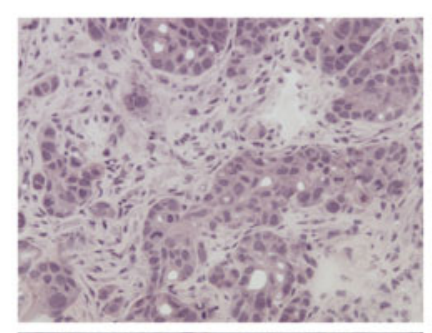

200x

$400 x$

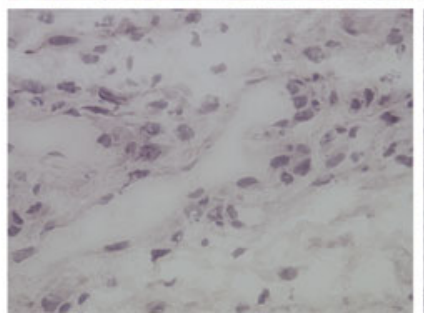

neg control
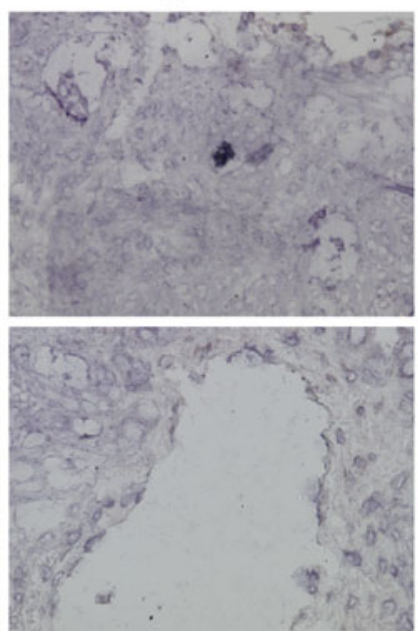

CD-31

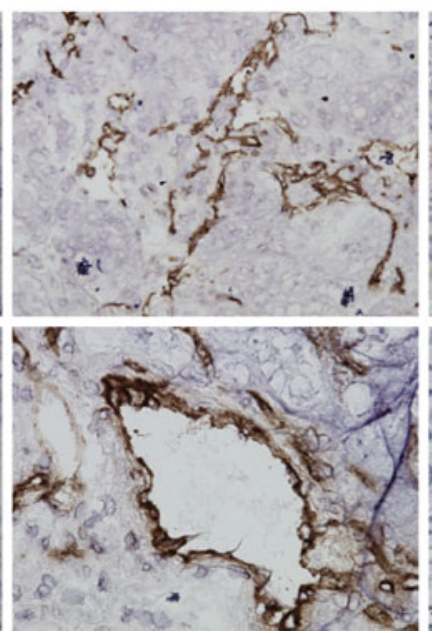

VEGFR2

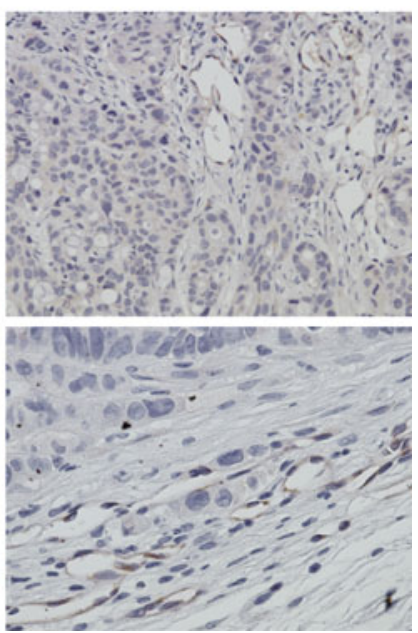

Fig. 4. Immunohistochemistry for CD-31 and VEGFR2. The peritumoral area shows distinct staining for CD-31 and weak staining for VEGFR2.

ultrasound using microbubbles targeted to VEGFR2 has been used in several preclinical studies [15-17]. Positron emission tomography or single photon emission computed tomography using radiotracers labeling VEGF or monoclonal antibodies specific to VEGFR2 has been shown to detect molecular targets defining vessels, but have the inconvenience of using radioactivity [13, 18, 19]. Fluorescent proteins and bioluminescence both are non-invasive, non-radioactive, and technically easy to handle [20]. Monoclonal antibodies labeled with fluorescence demonstrated differences in VEGFR2 expression [14]. In contrast to fluorescence, bioluminescence is more sensitive for imaging and lacks the background from autofluorescence [21]. Transgenic and knock-in animals expressing luciferase under the VEGFR2 promoter have been successfully used to visualize wound healing and peritumoral angiogenesis [22-24]. In these animal models, promoter activation/gene expression is measured by bioluminescence, rather than protein expression. In the present study, we used "knock-in" animals, which assure physiological levels of VEGFR2 promoter activation and protein expression.

The subcutaneous donor tumor induced VEGFR2 promoter activity only after growth to a certain size. In contrast, the implantation of the orthotopic tumor piece induced stronger VEGFR2 promoter activity. Performing a laparotomy creates a large area of wound healing, whereas a subcutaneous injection only induces small tissue damage. After the first period of wound healing, the light emission increased gradually to a second peak at 30 days and decreased thereafter. After growth to a certain size, tumors develop central necrosis, decreasing the mass of viable cells and thus the secretion of pro-angiogenic factors like VEGFA. This may be the reason for decreased VEGFR2 promoter activity and light emission after 30 days. But the level of light emission was still increased 4-fold compared to the background levels. Considering that bioluminescent light emission is being absorbed by the tissue, the same level of promoter activity results in a stronger light emission if the source is more superficial in the mouse. Tumor removal at sacrifice resulted in light emission at background levels for the mouse. This argues for the removal of the specific tumor-associated VEGFR2 promoter activity by explanting the tumors and the skin. The covering skin emitted only low levels of light, confirming the completion of wound healing. The highest light emission was found peritumorally. To confirm angiogenesis, we stained the tumor tissue for CD-31 and VEGFR2. VEGFR2 expression was not present in all the CD-31-positive vessels and was mainly expressed in peritumoral vessels. This is consistent with VEGFR2 acting in concert with VEGF-A as promoters of neoangiogenesis, but not as sustainer of vessel structures once the vessels are formed. As the present study was conducted to evaluate the feasibility to image angiogenesis in the VEGFR2-luc-KI mouse, the interpretation of the histological data is limited. A larger, subsequent study will be required to assess the quantitative correlation between bioluminescent light emission and VEGFR2 expression.

Pancreatic cancer is characterized by a strong desmoplastic reaction that drives the expression of factors responsive to hypoxia including VEGF-A, inducing the formation of new vessels. Preclinical studies have found that targeting cancer-derived angiogenesis, specifically VEGF, can slow the progression of pancreatic cancer in vivo [2528]. However, the application of this work in clinical trials has been disappointing to date [29]. While VEGF is a prime angiogenic factor, it is increasingly apparent that other angiogenic factors binding to different receptors contribute to the overall angiogenic cancer milieu. Therefore, a preclinical model visualizing angiogenesis is valuable to test new targets. VEGFR2-luc-KI mice are available under a C57/BL6 background. This enables the exploration of regulatory mechanisms in tumor angiogenesis by using various genetically modified mice. Future studies are warranted to demonstrate whether this promising approach 
is suitable to detect the angiogenic switch in transgenic mice of pancreatic carcinogenesis.

Acknowledgments. This work was supported by the UCLA in vivo Cellular and Molecular Imaging Center, the National Institutes of Health (P01 AT003960 and R21 CA124609), the Swiss National Science Foundation (PBZHB-117008), and the Hirshberg Foundation for Pancreatic Cancer Research. In vivo imaging was performed at the Crump Institute for molecular Imaging at UCLA. We thank David Stout and Harvey R. Herschman, Ralph and Marjorie Crump Endowed Chair in Molecular Imaging, for their advice.

Open Access. This article is distributed under the terms of the Creative Commons Attribution Noncommercial License which permits any noncommercial use, distribution, and reproduction in any medium, provided the original author(s) and source are credited.

\section{References}

1. Folkman J (2007) Angiogenesis: an organizing principle for drug discovery? Nat Rev Drug Discov 6:273-286

2. Naumov GN, Akslen LA, Folkman J (2006) Role of angiogenesis in human tumor dormancy: animal models of the angiogenic switch. Cell Cycle 5:1779-1787

3. Matsumoto T, Claesson-Welsh L (2001) VEGF receptor signal transduction Sci STKE 2001: RE21

4. Shibuya M, Claesson-Welsh L (2006) Signal transduction by VEGF receptors in regulation of angiogenesis and lymphangiogenesis. Exp Cell Res 312:549-560

5. Shalaby F, Rossant J, Yamaguchi TP et al (1995) Failure of bloodisland formation and vasculogenesis in Flk-1-deficient mice. Nature 376:62-66

6. Hatva E, Kaipainen A, Mentula P et al (1995) Expression of endothelial cell-specific receptor tyrosine kinases and growth factors in human brain tumors. Am J Pathol 146:368-378

7. Rousseau S, Houle F, Kotanides H et al (2000) Vascular endothelial growth factor (VEGF)-driven actin-based motility is mediated by VEGFR2 and requires concerted activation of stress-activated protein kinase 2 (SAPK2/p38) and geldanamycin-sensitive phosphorylation of focal adhesion kinase. J Biol Chem 275:10661-10672

8. Holmes K, Roberts OL, Thomas AM, Cross MJ (2007) Vascular endothelial growth factor receptor-2: structure, function, intracellular signalling and therapeutic inhibition. Cell Signal 19:2003-2012

9. Eibl G, Reber HA (2005) A xenograft nude mouse model for perineural invasion and recurrence in pancreatic cancer. Pancreas 31:258-262

10. Korpelainen EI, Karkkainen MJ, Tenhunen A et al (1998) Overexpression of VEGF in testis and epididymis causes infertility in transgenic mice: evidence for nonendothelial targets for VEGF. J Cell Biol 143:1705-1712

11. Aucejo F, Kim R, Zein N et al (2009) Vascular endothelial growth factor receptor 2 expression in non-tumorous cirrhotic liver is higher when hepatoma is beyond Milan criteria. Liver Transplant 15:169-176

12. Fukumura D, Jain RK (2008) Imaging angiogenesis and the microenvironment. Apmis 116:695-715
13. Willmann JK, Chen K, Wang $\mathrm{H}$ et al (2008) Monitoring of the biological response to murine hindlimb ischemia with $64 \mathrm{Cu}$-labeled vascular endothelial growth factor-121 positron emission tomography. Circulation 117:915-922

14. Virostko J, Xie J, Hallahan DE, Arteaga CL, Gore JC, Manning HC (2009) A molecular imaging paradigm to rapidly profile response to angiogenesis-directed therapy in small animals. Mol Imaging Biol 11:204-212

15. Korpanty G, Carbon JG, Grayburn PA, Fleming JB, Brekken RA (2007) Monitoring response to anticancer therapy by targeting microbubbles to tumor vasculature. Clin Cancer Res 13:323-230

16. Palmowski M, Huppert J, Ladewig G et al (2008) Molecular profiling of angiogenesis with targeted ultrasound imaging: early assessment of antiangiogenic therapy effects. Mol Cancer Ther 7:101-109

17. Loveless ME, Whisenant JG, Wilson K et al (2009) Coregistration of ultrasonography and magnetic resonance imaging with a preliminary investigation of the spatial colocalization of vascular endothelial growth factor receptor 2 expression and tumor perfusion in a murine tumor model. Mol Imaging 8:187-198

18. Choe YS, Lee KH (2007) Targeted in vivo imaging of angiogenesis: present status and perspectives Curr Pharm Des 13:17-31

19. Willmann JK, Cheng Z, Davis C et al (2008) Targeted microbubbles for imaging tumor angiogenesis: assessment of whole-body biodistribution with dynamic micro-PET in mice. Radiology 249: 212-219

20. Amoh Y, Katsuoka K, Hoffman RM (2008) Color-coded fluorescent protein imaging of angiogenesis: the AngioMouse models. Curr Pharm Des 14:3810-3819

21. Troy T, Jekic-McMullen D, Sambucetti L, Rice B (2004) Quantitative comparison of the sensitivity of detection of fluorescent and bioluminescent reporters in animal models. Mol Imaging 3:9-23

22. Zhang N, Fang Z, Contag PR, Purchio AF, West DB (2004) Tracking angiogenesis induced by skin wounding and contact hypersensitivity using a Vegfr2-luciferase transgenic mouse. Blood 103:617-626

23. Lyons S, Clermont $A$, Neben $T$ et al (2005) Non-invasive in vivo bioluminescent imaging of tumor angiogenesis in mice. AACR 96th Annual Meeting

24. Lyons S, Lim E, Chen $M$ et al (2006) Non-invasive in vivo bioluminescent imaging of tumor angiogenesis in mice. Society for Molecular Imaging - 5th Annual Meeting

25. Hotz HG, Hines OJ, Masood R et al (2005) VEGF antisense therapy inhibits tumor growth and improves survival in experimental pancreatic cancer. Surgery 137:192-199

26. Buchler P, Reber HA, Buchler M et al (2003) Hypoxia-inducible factor 1 regulates vascular endothelial growth factor expression in human pancreatic cancer. Pancreas 26:56-64

27. Duffy JP, Eibl G, Reber HA, Hines OJ (2003) Influence of hypoxia and neoangiogenesis on the growth of pancreatic cancer. Mol Cancer 2:12

28. Buchler P, Reber HA, Buchler MW, Friess H, Lavey RS, Hines OJ (2004) Antiangiogenic activity of genistein in pancreatic carcinoma cells is mediated by the inhibition of hypoxia-inducible factor- 1 and the down-regulation of VEGF gene expression. Cancer 100:201-210

29. Kindler HL, Friberg G, Singh DA et al (2005) Phase II trial of bevacizumab plus gemcitabine in patients with advanced pancreatic cancer. J Clin Oncol 23:8033-8040 